\title{
MODELING OF THE DYNAMIC LOADS OF THE HUMAN STOMATOGNATHIC SYSTEM DURING BITING OFF
}

\author{
P. Stróżyk ${ }^{*}$, J. Bałchanowski** ${ }^{* *}$ J. Szrek ${ }^{* * * *}$
}

\begin{abstract}
In physiological conditions, food is a factor having a significant bearing on the values of the loads acting on the stomatognathic system. On the basis of the mechanical properties of the food the brain coordinates and regulates the activity of the muscles in such a way that they generate a proper bite force adjusted to the foodstuff being eaten. The investigations were divided into two stages: 1) experimental tests and 2) numerical simulations. In the first stage, classic force-displacement characteristic curves were determined for the food while in the second stage, the curves were used as a dynamic stomatognathic system model load function. One of the most important results of this research is the proof that there is a full dependence between the food and the muscular forces and the temporomandibular joint (TMJ) reactions.
\end{abstract}

Keywords: dynamic model of stomatognathic system, forces in muscles of mastication, responses in temporomandibular joint

\section{Introduction}

The human stomatognathic system is a typical example of a kinematically and mechanically indeterminate system. Owing to the structure of the temporomandibular joint (TMJ) the human mandible is highly mobile relative to the cranium (Koolstra and van Eijden, 2001, 2005). In each of the joints the mandibular head is guided on the articular tubercle of the temporal bone. Thanks to this mandible-skull articulation both the hinge (rotary) movement and the gliding (translational) movement can be performed. Assuming that the bones in the joints are ideally rigid (undeformable) and that during the movement of the mandible the contact between the bones is constantly assured, this articulation provides four degrees of freedom.

The primary stage in the creation of a human stomatognathic system model consists in defining: the number of active muscles (forces acting on the mandible bone) needed to prepare a proper mandible loading case (Koolstra and van Eijden, 2005; Osborn and Baragar, 1985; Stróżyk, 2016; van Essen et al., 2005) i.e. biting off or mastication.

An analysis of the mandible dynamics indicates that in physiological conditions, the heaviest loads occur when eating meals. Hence, first of all the muscles of mastication should be taken into account in the loading configuration. Food is a factor having a significant effect on the values of the loads acting on the mandible (Woda et al., 2006).

On the basis of a biochemical analysis it was decided that the main goal would be to determine the forces (muscular forces and TMJ reactions) acting on the stomatognathic system during biting off depending on the selected food (the force-displacement characteristic).

Assis. Prof. Przemysław Stróżyk, PhD.: Wroclaw University of Science and Technology, Faculty of Mechanical Engineering, Łukasiewicza Street 5; 50-371 Wrocław, PL, przemyslaw.strozyk@pwr.edu.pl

** Assoc. Prof. Jacek Bałchanowski, DSc., PhD.: Wroclaw University of Science and Technology, Faculty of Mechanical Engineering, Łukasiewicza Street 5; 50-371 Wrocław, PL, jacek.balchanowski @pwr.edu.pl

** Assis. Prof. Jarosław Szrek, PhD.: Wroclaw University of Science and Technology, Faculty of Mechanical Engineering, Łukasiewicza Street 5; 50-371 Wrocław, PL, jaroslaw.szrek@pwr.edu.pl 


\section{Methods}

In order to determine muscular force and TMJ reactions patterns depending on the selected food it was necessary to divide the computations into two stages. In the first stage, experimental tests were carried out and representative characteristic curves $\left(F^{s}-\Delta h^{s}\right.$, i.e. bite force versus change in food sample height) for the selected food was prepared. In the second stage, numerical simulation was carried out using a dynamic stomatognathic system model developed by the authors and characteristic curves $F^{s}-\Delta h^{s}$ as the load function.

\subsection{Experimental tests}

Special holders (Fig. 1a), holding the lower and upper incisors and the canines and ensuring their anatomically correct mutual alignment, had to be designed and made in order to determine the $F^{s}-\Delta h^{s}$ characteristic of foodstuff (Fig. 1b).

a)

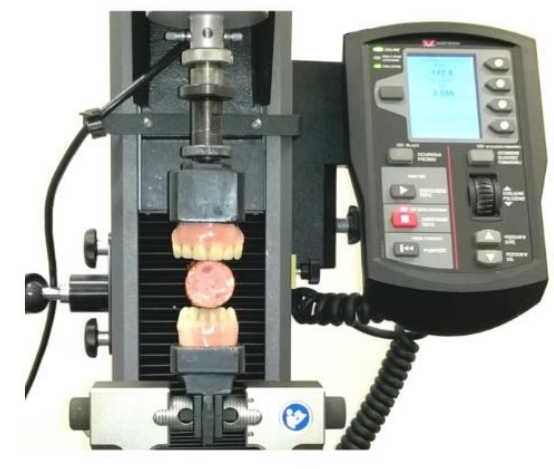

b)

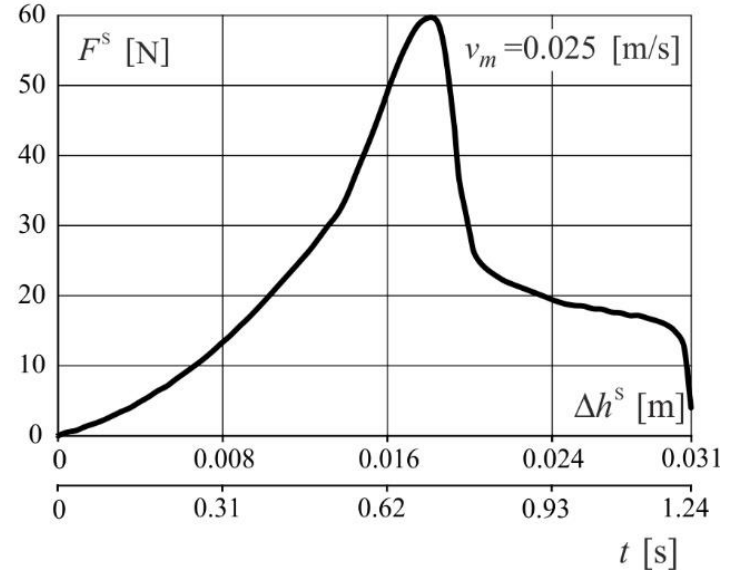

Fig. 1: Test setup: a) special holders and sausage sample, b) representative characteristic $F^{s}-\Delta h^{s}$

The experimental tests were based on the following assumptions: 1) the rate of biting off was constant, amounting to $v_{m}=0.025 \mathrm{~m} / \mathrm{s}$ (Wang and Stohler, 1991), 2) when selecting food it was assumed that had to assure rotation with simultaneous translation in TMJ, 3) the $F^{s}-\Delta h^{s}$ curve for the selected foodstuff were determined (statistically) on the basis of $F-\Delta h$ (single sample), 4) for technical reasons no saliva. 18 sausage samples (mean height $\pm \mathrm{SD} h_{s}=0.031 \pm 0.002 \mathrm{~m}$ ) were prepared for experimental tests. The experiments were carried out using an Instron 5944 (Bluehill ver. 3) strength testing machine.

\subsection{Building of numerical model of the human stomatognathic system}

The numerical model of the stomatognathic system was based on the geometry of solid models of the human skull and mandible (Synbone). The numerical model comprised the maxilla, the mandible, the two TMJ joints, the temporal muscle forces $F_{\mathrm{r}}^{\mathrm{T}}$ and $F_{1}^{\mathrm{T}}$, the masseter muscle forces $F_{\mathrm{r}}^{\mathrm{M}}$ and $F_{1}^{\mathrm{M}}$ and the medial pterygoid muscle forces $F_{\mathrm{r}}^{\mathrm{MP}}$ and $F_{1}^{\mathrm{MP}}$ (Fig. 2a). The muscle attachment points were adopted based on the geometry described in the articles (Sinelnikov,. 1988; Stróżyk and Bałchanowski, 2016). A parametric model of the food being bitten off as a function of force $F^{\mathrm{s}}$ (Fig. 1b) was placed between the incisors (between the points $\mathrm{C}_{\mathrm{L}}$ and $\mathrm{C}_{\mathrm{H}}$ ) in the model as the external load (Fig. 2).

On the basis of an analysis of the structure and biomechanics the TMJ joints were modelled as two shape joints, with constraints in the form of contact forces interactions between the articular surface of the mandibular condyle and the articular tubercle of the temporal bone (Fig. 2). The proposed articulation model enables the simulation of the complex movement of the lower incisors (rotation and gliding) during biting off. Every shape joint between the mandible and the skull formed in this way is a $\mathrm{V}$ class pair (with five degrees of freedom). In such case the whole of model has 4 DoF. It means that four unknown active muscular forces could be determined from the equations of equilibrium. Hence two temporal muscle forces: $F_{\mathrm{r}}^{\mathrm{T}}$ and $F_{1}^{\mathrm{T}}$ were assumed to be the unknowns in the model while the masseter forces $F_{\mathrm{r}}^{\mathrm{M}}$ and $F_{1}^{\mathrm{M}}$ and the medial pterygoid forces $F_{\mathrm{r}}^{\mathrm{MP}}$ and $F_{1}^{\mathrm{MP}}$ were replaced with two unknowns resultant forces $F_{\mathrm{r}}^{\mathrm{V}}$ and $F_{1}^{\mathrm{V}}$ (Stróżyk, 2016) on the left and right side of the mandible (Fig. 2b). 

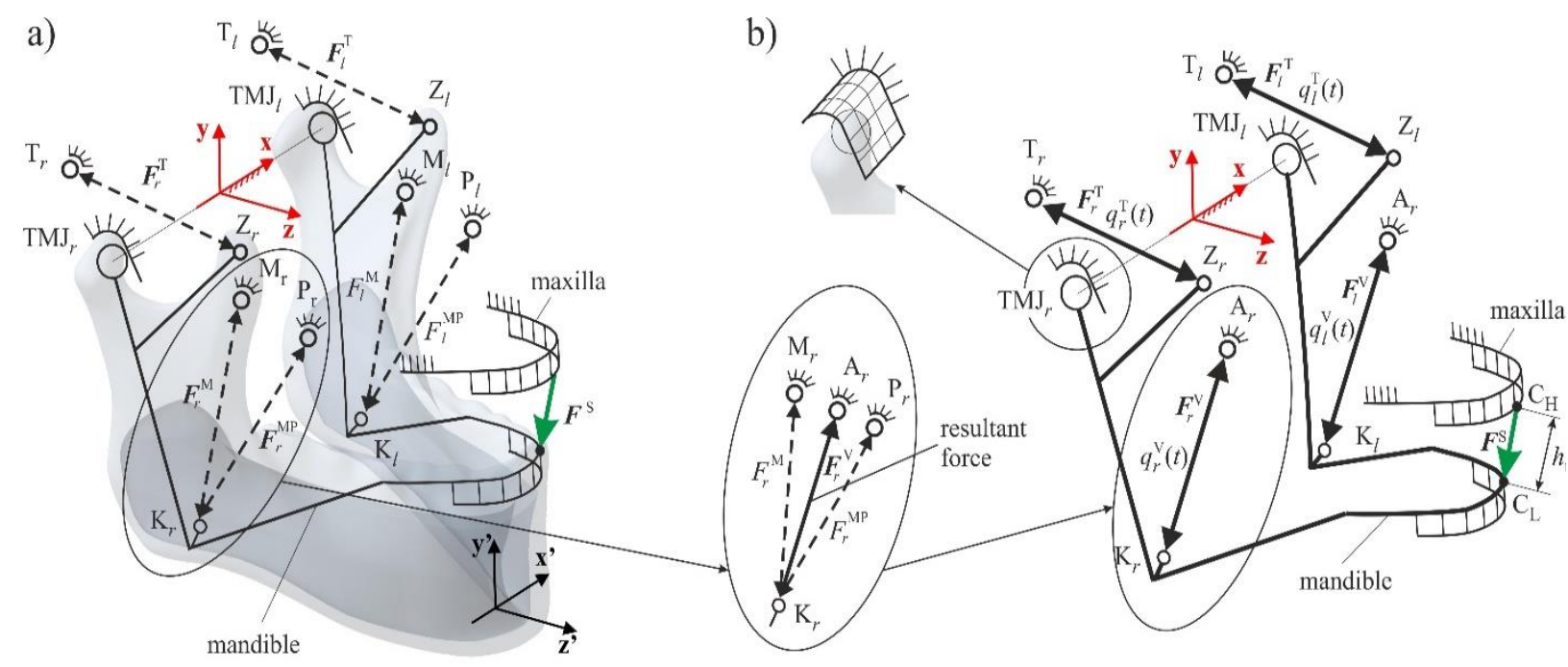

Fig. 2: Model of human stomatognathic system: a) scheme with mandibular elevator muscles forces, b) numerical model

Four kinematic excitations: $q_{1}^{\mathrm{T}}(t), q_{\mathrm{r}}^{\mathrm{T}}(t), q_{1}^{\mathrm{V}}(t)$ and $\mathrm{q}_{\mathrm{r}}^{\mathrm{V}}(t)$ were adopted in the numerical stomatognathic system model. The excitations had the form of linear drives enabling the movement of the mandible relative to the skull (Fig. 2b) and modelling the expansion and contraction of the mandibular elevator muscles. The runs of $q_{1}^{\mathrm{T}}, q_{\mathrm{r}}^{\mathrm{T}}, q_{1}^{\mathrm{V}}$ and $q_{r}^{\mathrm{V}}$ excitations were determined by modeling and solving the inverse task of kinematics of the stomatognathic system for given motion of incisors. Determined courses of these kinematic excitations are presented in the Fig. 3. Active forces $F_{1}^{\mathrm{T}}$ and $\mathrm{F}_{\mathrm{r}}^{\mathrm{T}}$ in excitations $q_{1}^{\mathrm{T}}(t)$ and $q_{\mathrm{r}}^{\mathrm{T}}(t)$ represent the temporalis forces while active forces $F_{1}^{\mathrm{V}}$ and $F_{\mathrm{r}}^{\mathrm{V}}$ in excitations $q_{1}^{\mathrm{V}}(t)$ and $q_{\mathrm{r}}^{\mathrm{V}}(t)$ represent the resultant forces of the masseter forces and the medial pterygoid forces. Active forces would be determine in simulation.

\subsection{Simulation studies of process of biting off food model}

In the simulations the mandible would be moved from the lower position until the upper and lower teeth touched (clenched). General scheme of simulation is presented in Fig. 2b. Height $h_{\mathrm{t}}$ stands for the distance between the upper and lower incisors (distance between points $\mathrm{C}_{\mathrm{L}}$ and $\mathrm{C}_{\mathrm{H}}$ ).

The food force $F^{s}$ curve used as the dynamic stomatognathic system load function. The incisors were positioned at distance $h_{\mathrm{t}}=h_{\mathrm{s}}=0.031 \mathrm{~m}$ in the initial position (at time $t=0 \mathrm{~s}$ ). Which means that in the simulation starting point, the teeth were in contact with the food. The simulation was continued until the contact between the upper and lower incisors was reached $\left(h_{\mathrm{t}}=0\right)$. During the simulation of biting off food the incisors would move at constant velocity $v_{m}$.

The solid computational model was used to run numerical dynamic simulations of biting off in the LMS Dads ver. 9.6 (www.plm.automation.siemens.com, 2018) computer system for the dynamic analysis of multibody systems (Haug, 1989).

\section{Results}

Based on the simulation, two temporal muscle forces $F_{\mathrm{r}}^{\mathrm{T}}$ and $F_{1}^{\mathrm{T}}$, two resultant forces $F_{\mathrm{r}}^{\mathrm{V}}$ and $F_{1}^{\mathrm{V}}$ and two reactions forces $F_{\mathrm{r}}^{\mathrm{TMJ}}$ and $F_{1}^{\mathrm{TMJ}}$ in TMJ joints were determined for stomatognathic system loaded by given food force $F^{\mathrm{s}}$. The resultant force $F_{\mathrm{r}}^{\mathrm{V}}$ and $F_{1}^{\mathrm{V}}$ were divided into components: on the two masseter forces $\left(F_{\mathrm{r}}^{\mathrm{M}}\right.$ and $\left.F_{1}^{\mathrm{M}}\right)$ and the two medial pterygoid forces $\left(F_{\mathrm{r}}^{\mathrm{MP}}\right.$ and $\left.F_{1}^{\mathrm{MP}}\right)$. The simulations yielded the results are presented as a function of parameter $h_{\mathrm{t}}$ or $\Delta h^{\mathrm{s}}$ in Fig. 4. Owing to the symmetry (the model geometry, the muscular system and the load) of the stomatognathic system, the values of forces were identical for left and right side of model and so only changes in force for right side are shown in the diagrams (Fig. 4). 


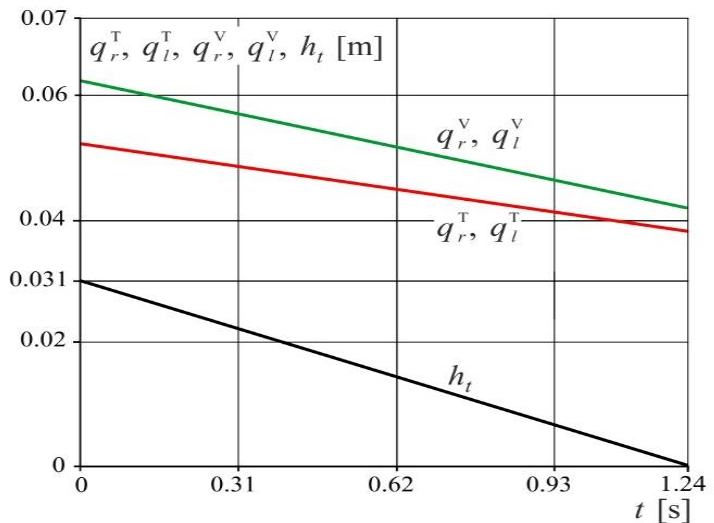

Fig. 3: Kinematic excitations $q_{r}^{V}, q_{l}^{V}, q_{r}^{T}, q_{l}^{T}$ and displacements of incisors $h_{t}$

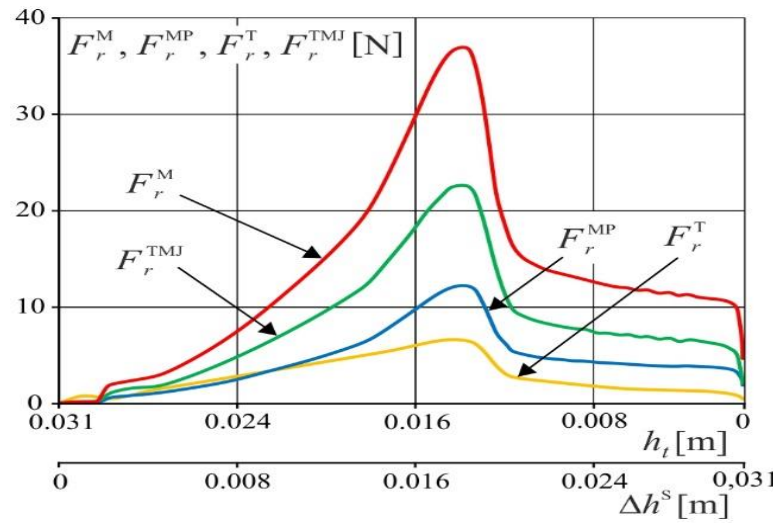

Fig. 4: Muscular force $F_{r}^{M}, F_{r}^{M P}, F_{r}^{T}$ and TMJ reactions $F_{r}^{T M J}$

\section{Conclusions}

One of the most important results of this research is that it has shown that there is a full dependence between food and the muscular forces and the TMJ reactions. An analysis of the results showed that the muscular force and TMJ reactions were synchronized with the characteristics of foodstuff $\left(F^{s}-\Delta h^{s}\right)$, i.e. the system's response was consistent with the movement forcing function. The results shown in Fig.4 that the masseter and the medial pterygoid contribute most to the generation of the maximum bite force, while the temporalis contributes least. The reaction forces in TMJ are definitely greater than the forces in the medial pterygoid and the temporalis. The dependencies partially corresponds to the results presented in (Throckmorton et al., 1990; Farella et al., 2008, Bałchanowski and Stróżyk, 2016) while the muscular force values are consistent with the ones reported in (Reina et al., 2007), especially for the masseter and the temporalis.

The simulation studies were prepared only for one selected food - sausage but a parametric model of the food being bitten off is general. By changing the parameters of the food model wide groups of foodstuffs can be analysed.

\section{References}

Farella M., Palla S., Erni S., Michelotti A. and Gallo L.M. (2008) Masticatory muscle activity during deliberately performed oral tasks. Physiol. Meas. 29, 1397-1410.

Haug E. J. (1989) Computer Aided Kinematics and Dynamics of Mechanical Systems. Allyn and Bacon, Boston.

Koolstra J. and van Eijden T. (2001) A method to predict muscle control in the kinematically and mechanically indeterminate human masticatory system. J. Biomech. 34, 1179-1188.

Koolstra J. and van Eijden T. (2005) Combined finite-element and rigid-body analysis of human jaw joint dynamics. J. Biomech. 38, 2431-2439.

Osborn J. W. and Baragar F. A. (1985) Predicted pattern of human muscle activity during clenching derived from a computer assisted model: symmetric vertical bite forces. J. Biomech. 18, 599-612.

Reina J.M., Garcia-Aznar J.M., Dominguez J. and Doblaré M. (2007) Numerical estimation of bone density and elastic constants distribution in a human mandible. J. Biomech. 40, 826-836.

Sinelnikov R. (1988) Atlas of human anatomy, Mir, Moscow.

Stróżyk P. (2016) Evaluation of forces in elevator muscles of mandible. Scholar's Press.

Stróżyk P., Bałchanowski J. (2016) Effect of foodstuff on muscle forces during biting off. Acta Bioeng. Biomech. $18,81-91$.

Throckmorton G.S., Groshan G.J. and Boyd S.B. (1990) Muscle activity patterns and control of temporomandibular joint loads. J. Prosthet. Dent. 63, 685-695.

van Essen, N.L., Anderson, I.A., Hunter, P.J., Carman, J.B., Clarke, R.D. and Pullan, A.J. (2005) Anatomically based modelling of the human skull and jaw. Cells Tissues Organs. 180, 44-53.

Wang J. S. and Stohler C, S. (1991) Predicting foodstuff from jaw dynamics during masticatory crushing in man. Arch. Oral Biol. 36, 239-244.

Woda A., Foster K., Mishellany A. and Peyron M.A. (2006) Adaptation of healthy mastication to factors pertaining to the individual or to the food. Physiol. Behav. 89, 28-35.

https://www.plm.automation.siemens.com/en/products/lms/virtual-lab/legacy-applications/dads.shtml (20.03.2018) 